\title{
Lebenszyklus in Vereinsform tätigen Initiativen der Hilfe auf Gegenseitigkeit
}

In Seniorengenossenschaften werden traditionelle Formen der Solidarität wiederbelebt. Bürgerinnen und Bürger engagieren sich in Vereinsform auf der Basis der Hilfe auf Gegenseitigkeit. Das am Seminar für Sozialpolitik der Universität zu Köln durchgeführte und von der Robert Bosch Stiftung finanzierte Forschungsprojekt „Motive und Handlungsmuster von Bürgern in Seniorengenossenschaften "l analysiert mit seinem biographischen Ansatz die Motivationsstrukturen der Mitglieder von Seniorengenossenschaften, zusätzlich werden Einblicke in den Lebenszyklus von Seniorengenossenschaften offen gelegt. Der folgende kurze Beitrag diskutiert den Lebenszyklus von Initiativen der Hilfe auf Gegenseitigkeit, die unter dem Begriff Seniorengenossenschaft subsumiert werden.

Mit seinem biographischen Ansatz stellt das am Seminar für Sozialpolitik durchgeführte Forschungsprojekt Motive und Handlungsmuster von Bürgern in Seniorengenossenschaften das Subjekt selbst, die interviewte Person in den Mittelpunkt. Das Subjekt schafft sich die sinnhafte Strukturierung seines sozialen Handelns und baut ein ihm eigenes Selbstund Weltbild auf. Dabei verweilt das Forschungsprojekt nicht auf der Mikroebene, sondern deckt auch Strukturen der Mesoebene auf. ${ }^{2}$ Auf der Mesoebene ist eine Seniorengenossenschaft als eine Gemeinschaft zu verstehen, deren Fundament der gelebte Gegenseitigkeitsgedanke bildet. ${ }^{3}$ Auf freiwilliger Basis wird in Vereinsform ${ }^{4}$ eine ursprüngliche Hilfeform der gegenseitigen Unterstützung gewährt sowie Gesellung erfahren; dabei dienen Zeitkonten als Verrechungssystem für geleistete und erhaltene Hilfen. Die Mitglieder leben eine Gemeinschaft, die vom Geben und Nehmen (Mauss 2007) der Mitglieder getragen und deren Stabilität vom Zusammenspiel der einzelnen Akteure bestimmt wird. In diesem Sinne sind die Biographien der Seniorengenossenschaftler untereinander verstrickt. Je nach Mitgliederstärke einer Seniorengenossenschaft setzt sich die Gemeinschaft aus einer Vielzahl von Gruppen zusammen, die in ihrer Dynamik stabilisierend, mitunter aber auch destabilisierend wirken. Oft entwickeln die Mitglieder ein Zugehörig-

1 Endbericht in: Köstler/Schulz-Nieswandt 2009. Genossenschaftliche Selbsthilfe von Senioren. Motive und Handlungsmuster bürgerschaftlichen Engagements, Stuttgart.

2 Nachfolgende Überlegungen basieren auf der qualitativen Befragung von 20 Mitgliedern einer Seniorengenossenschaft im Rahmen des hier vorgestellten Forschungsprojekts Motive und Handlungsmuster von Bürgern in Seniorengenossenschaften sowie auf den Ergebnissen des ebenfalls am Seminar für Sozialpolitik in den Jahren 2003-2006 durchgeführten quantitativen Forschungsprojekts Seniorengenossenschaften - Stabilitätseigenschaften und Entwicklungschancen.

3 Seniorengenossenschaften sind moderne Erscheinungen der 1990er Jahre, die dem Ansatz der Selbsthilfe folgen. Das Handeln der Mitglieder von Seniorengenossenschaften wird in einer gabebasierten Motivation fundiert, die die dort gelebte generalisierte Reziprozität stabilisiert. Gelebt wird - wie Marcel Mauss es theoretisierte - ein Geben, Nehmen und Erwidern, wobei bei den Seniorengenossenschaften die Gegengabe eine Option ist, die in der Zukunft eingelöst, dann Reziprozität auslöst. Vgl. Köstler 2006.

4 Als Rechtsform wählen die Seniorengenossenschaften den eingetragenen Verein. 
keitsgefühl zu der Gruppe (Museumsgruppe, Wandergruppe, PC-Gruppe, Gesprächsgruppe), der sie sich anschließen und die sie mit ihren Ideen sowie durch ihre Teilhabe am Gruppengeschehen gestalten. Dieses Identifikationserlebnis der einzelnen Mitglieder wird auf den Verein transportiert. So bekommt auch die Seniorengenossenschaft als Institution eine Biographie, die durch das Wirken der Mitglieder über die bisherigen Vereinsjahre erzeugt wird.

\section{Die Idee Seniorengenossenschaft}

Die ersten Seniorengenossenschaften gründeten sich Anfang der 1990er Jahre im Rahmen des von der Baden-Württembergischen Landesregierung geförderten Modellprogramm Seniorengenossenschaften. Weitere Seniorengenossenschaften folgten Mitte der 1990er Jahre in Hessen im Kreis Offenbach, ebenfalls begleitet von einem Förderprogramm. Das Konzept des Kreises Offenbach hatte eine bedeutende regionale Multiplikatorwirkung, derart dass sich in der Region ca. weitere 30 Seniorengenossenschaften etablierten, zusätzlich gründeten sich an vereinzelten Standorten im Bundesgebiet weitere Seniorengenossenschaften. Derzeit initiiert die Ehrenamtsagentur des Kreises Offenbach weitere Initiativen der Hilfe auf Gegenseitigkeit in Nordhessen. Eine amtliche Statistik gibt es nicht, es kann aber davon ausgegangen werden, dass es aktuell im Bundesgebiet etwa 60 Vereine der Hilfe auf Gegenseitigkeit, einzuordnen als Initiativen des Dritten Sektors (Schulz-Nieswandt 2008), gibt. Gemeinsam ist diesen in Vereinsform tätigen Initiativen, dass sie ein mitgliederorganisiertes Angebot an Hilfen im Alltag, den so genannten $\mathrm{IADL}^{5}$, anbieten, für ihre Mitglieder zahlreiche Veranstaltungen der Gesellung organisieren und auf der Basis von Zeitgutscheinen arbeiten. Die Angebotspalette der Initiativen zielt in die Lücke zwischen dem Leitungsangebot der Gesetzlichen Pflegeversicherung und marktfähigen professionell angebotenen Dienstleistungen (Handwerk, Beratung, Personenbeförderung). ${ }^{6}$ So fangen die Unterstützungshilfen der Seniorengenossenschaften dort an, wo die mit stark reguliertem Leistungskatalog ausgestattete, auf die ADLs konzentrierte Gesetzliche Pflegeversicherung nicht mehr tätig wird (eben bei Hilfen im Haushalt, Hilfen bei Behörden, allgemein: Hilfen zur Teilhabe am sozialen Leben) und wo ein tragendes Netzwerk fehlt, da Verwandtschaft mangels Zeit oder aus räumlicher Entfernung nicht tätig werden kann oder will und nachbarschaftliche Strukturen nicht mobilisiert werden können.

Gerade das Zeitkontensystem, anlehnend an das in Tauschringen ${ }^{7}$ praktizierte LETSystem, stellt für die Initiativen einen stabilisierenden Faktor dar. Allerdings schreiben Seniorengenossenschaften ausschließlich positive Zeitpunkte gut und das Ansparen von

5 Abgegrenzt von den ADL (Activities of Daily Living), die den Leistungsrahmen der gesetzlichen Pflegeversicherung abstecken, sind IADL (Instrumental Activities of Daily Living) instrumentelle Aktivitäten des täglichen Lebens, die nach Lawton und Brody Tätigkeiten wie Haushaltsführung, Einkaufen, Nutzung von Verkehrsmitteln u.a. umfassen und somit allgemein zur Teilhabe am sozialen Leben notwendig sind.

6 Somit weisen Seniorengenossenschaften Charakterzüge von Selbsthilfegruppen auf, obwohl sie nicht unter die Förderung des § 20 c SGB V fallen. Dazu: Schmale/Blome-Drees 2006.

7 Zu Tauschringen: Wagner 2008. 
Zeitpunkten für die Zukunft ist ausdrücklich gewünscht. ${ }^{8}$ Das System Seniorengenossenschaft lebt davon, dass die Mitglieder heute Hilfen geben und auf die in die Zukunft gerichtete Gegenleistung an zu erhaltenen Hilfen vertrauen. So kann die Bereitschaft der Mitglieder zum Gabeüberschuss als fundamental für die Idee Seniorengenossenschaft erachtet werden (Schulz-Nieswandt 2006a).

Die ersten Seniorengenossenschaften blicken derzeit auf eine bis zu 17jährige Vereinsgeschichte zurück. Die Initiativen haben in diesen Vereinsjahren verschiedene Phasen durchlebt. Die Mitglieder haben durch ihr Engagement den Verein geprägt und es zeigt sich, da das Vereinsangebot mitgliederbestimmt ist, dass jede Seniorengenossenschaft eine eigene Biographie hat. Bei aller mitgliederbezogenen Prägung der Vereine zeigen sich jedoch verallgemeinerbare Vereinsphasen, die jede Seniorengenossenschaft durchlebt, wenn auch unterschiedlich ausgeprägt und in unterschiedlicher zeitlicher Dauer. Nach der Gründungsphase folgt ein Zeitabschnitt, in dem sich der Verein in das Vereinsbild der Region etabliert, anschließend muss sich der Verein festigen, damit mögliche Instabilitäten, hervorgerufen durch personalen Wechsel im Vorstand und/oder der Kerngruppe des Vereins sowie in Form von Veränderungen der regionalen Einbettung (Personalwechsel bei den Behörden und anderen Vereinen in der Region, Etablierung weiterer auf dem Gebiet des Sozialen tätiger Vereine) bewältigt werden können.

\section{Gründungsphase}

Die ersten beiden Vereinsjahre sind vom Elan der Gründungsmitglieder bestimmt. Es sind vor allem als „Sinn suchende Idealisten“ zu bezeichnende Persönlichkeiten, die die Gründung der Seniorengenossenschaft initiieren und vorantreiben. Diese Personen haben einen inneren Drang, Neues zu schaffen. Angetrieben werden sie von einer Suche nach etwas im Leben Verlorenen, dem sie sich nach wie vor verbunden fühlen, das sie aber aus unterschiedlichen Gründen nicht mehr erreichen können oder wollen. In der Idee Seniorengenossenschaft wird die Möglichkeit gesehen, Sehnsüchte nach der verlorenen Heimat, nach der in der kindlichen Erinnerung als tragend empfundenen Dorfgemeinschaft und Nachbarschaft, nach der im Lebenszyklus verloren gegangenen familiären Einbettung oder der mit dem Renteneintritt beendeten beruflichen Verkettung zu leben (SchulzNieswandt 2006). Wichtig in dieser Gründungsphase ist, dass es die Initiatoren der Seniorengenossenschaft schaffen, den Verein auf eine breite Mitgliederbasis zu stellen. Vor allem aktive, sich in die Gesellungsgruppen einbringende und Hilfeleistungen anbietende Mitglieder gilt es an den Verein zu binden. ${ }^{9}$ Dabei erfüllen die Gründer der Seniorengenossenschaften großteils die einem social entrepreneur zugeschriebenen Eigenschaften. ${ }^{10}$

8 Zum Vergleich zwischen Seniorengenossenschaften und Tauschringen: Köstler 2007.

9 Im Durchschnitt nimmt ein Viertel der Mitglieder aktiv am Vereinsleben teil, mit unterschiedlicher Frequenz, manche besuchen wöchentliche Veranstaltungen, andere haben nur zweimal im Jahr Kontakt mit der Initiative. Die übrigen drei Viertel sind passive Mitglieder, zahlen den Jahresbeitrag, partizipieren aber nicht mehr oder noch nicht am Vereinsleben.

10 Eine kurze Übersicht über die Social Entrepreneurship-Ansätze in: Beyes/Jäger 2005. 
Ziel in der Gründungsphase ist es, eine ausreichend große Zahl Aktiver zu gewinnen. Hier sind es vor allem Personen, die in einer ereignisreichen, mitunter auch krisenbehafteten Lebenssituation bei den Vereinsangeboten Ablenkung suchen und finden. Da der Verein neu im Stadtbild ist und sich in den lokalen Medien schwerpunktmäßig mit dem Differenzierungsmerkmal des Zeitkontensystems präsentiert, werden auch Personen auf die Seniorengenossenschaft aufmerksam, für die gerade das Zeitkontensystem einen Anreiz zum Beitritt bietet. Diese werden aktiv und sammeln zielgerichtet Zeitpunkte, um sich gegen eine spätere Unterstützungsbedürftigkeit abzusichern. Auch kann der Verein mit seinem geringen Jahresbeitrag ${ }^{11}$ die Möglichkeit bieten, mit niedrigem finanziellen Aufwand die Freizeit abwechslungsreich zu gestalten. Mitunter können auch noch nicht vereinsmäßig engagierte Bürger zur Mobilisierung ihres externen Engagementpotentials veranlasst werden, da sie in der Idee der Seniorengenossenschaft eine Anreizstruktur zur Teilhabe entdecken. Ausschlaggebend ist, dass die Seniorengenossenschaft in der Gründungsphase auch Bürgerinnen und Bürger für ein Engagement gewinnt, die bereits in anderen Vereinen tätig sind oder die sich in der eigenen Familie intensiv engagieren und die bereit sind, ihr internes Engagementpotential auszuweiten. ${ }^{12}$

\section{Etablierungsphase}

In den Interviews ${ }^{13}$ schildern die Gründungsmitglieder eine geradezu euphorische Aufbruchsstimmung, die die Monate vor und nach der Gründungsversammlung der Seniorengenossenschaft prägen. Nach der Gründungsphase flacht diese antreibende Euphorie $a b$ und wird von den sich zwangsläufig ergebenen organisatorischen und administrativen Hürden eingeebnet. Der Vereinsalltag beginnt. Der Verein muss sich festigen und mit dem Vereinsbild der Stadt vernetzen. Zum erfolgreichen Bestehen dieser Etablierungsphase (etwa 2.-5. Vereinsjahr) ist es wichtig, dass ein Kontaktnetz zu anderen Institutionen vor Ort aufgebaut wird. Dabei spielen einzelne Mitglieder, die als Kerngruppe zu bezeichnen sind, eine tragende Rolle, indem sie ihre bestehenden Kontakte vor Ort nutzen, um den Verein zu etablieren. Entscheidend ist eine Abgrenzung zu anderen auf dem Gebiet des Sozialen tätigen Vereinen, insbesondere Konkurrenzsituationen müssen verhindert werden. Eine erfolgreiche Bewältigung dieser Etablierungsphase bedeutet, dass der Verein sein Angebot als ergänzend zum Angebot anderer auf dem Gebiet des Sozialen tätigen Vereinen etabliert. Dazu gehört auch der bewusste Verzicht auf Leistungen, die bereits erfolgreich und in ausreichendem Maße von anderen Vereinen im Stadtgebiet angeboten werden. Weiterhin sollte mit den städtischen Sozialstationen eine enge $\mathrm{Zu}$ sammenarbeit angestrebt werden, so dass parallele Angebote keine Konkurrenzsituation entstehen lassen. Entscheidend dabei ist, dass die Persönlichkeiten der Führungsebene

11 Der durchschnittliche jährliche Mitgliederbeitrag der 50 befragten Seniorengenossenschaften lag bei 12 Euro.

12 Der Freiwilligensurvey 1999-2004 konstatiert für das Jahr 2004 ein internes Engagementpotential von 32 \%. BFSFJ 2005.

13 Das Forschungsprojekt führte 20 narrativ-biographische Interviews, die durch einen leitfadengestützten Nachfrageteil ergänzt wurden. 
der Vereine bestrebt sind, ein kooperatives Nebeneinander der Vereine zu realisieren. Von großer Bedeutung ist ein direkter und von Zusammenarbeit getragener Kontakt zu den Verwaltungsbehörden der Stadt, um so die Mitarbeiter der Stadtverwaltung für die Unterstützung der Vereinsidee zu gewinnen. Ein cleverer Schachzug ist, schon in der Gründungsversammlung den Bürgermeister und in der Stadtlandschaft in Führungspositionen etablierte Persönlichkeiten als Mitglieder für den Verein zu gewinnen. Die Mitglieder der Kerngruppe, die das Netzwerk der Seniorengenossenschaft aufbauen und den Verein mit dem regionalen Vereinsbild vernetzen, stellen Sozialkapital in Form von bridging capital dar. Die Seniorengenossenschaftler stehen als Gruppenmitglieder den anderen Vereinen und Institutionen vor Ort gegenüber und bridging wird notwendig, um Verbundenheit herzustellen. Bei diesen Außenbeziehungen zu fremden Sozietäten steht dem bridging capital der Seniorengenossenschaft ein bridging capital der anderen vor Ort tätigen Sozietäten gegenüber (Patulny/Svendsen 2007).

In der Etablierungsphase treffen dann auch Personen auf die Seniorengenossenschaft, deren Vereinsaktivität als randständiges, freizeitorientiertes Gelegenheitsengagement bezeichnet werden kann. Diese Personen verfügen über internes oder aber - wenn noch in keiner weiteren Gemeinschaft (Familie, Nachbarschaft, Verein) aktiv - über externes Engagementpotential, das in der Regel durch Impulse von außen fremdangestoßen wird. Angesprochen von Vereinsmitgliedern lassen sie sich für ein Engagement in der Seniorengenossenschaft gewinnen. Oft sind in regelmäßigen Abständen motivierende Impulse nötig - auch in Form von persönlicher Ansprache -, damit die Aktivität in der Seniorengenossenschaft nicht zu einer passiven Mitgliedschaft wechselt. Seltener gehen diese Personen eigeninitiiert auf die Suche nach Engagementfeldern. Die Gründe für einen Eintritt in die Seniorengenossenschaft sind dabei äußerst vielschichtig. Es kann der Spaß, mit anderen etwas gemeinsam zu unternehmen, anderen helfen zu wollen, etc. sein. Wichtig ist, dass - unabhängig davon, ob eigen- oder fremdinitiiert - in der Seniorengenossenschaft ein persönlicher Berührungspunkt für die Aufnahme eines Engagements gefunden wird.

In der Etablierungsphase sind es dann auch wieder Personen in ereignisreichen oder gar krisenbehafteten Lebenssituationen, die auf den Verein stoßen und dort aktiv werden. Dabei kann der Eintritt in die Verrentung oder die Verrentung des Partners so ein Ereignis darstellen. Auch in Situationen der Pflege eines nahen Angehörigen kann Ablenkung gefunden werden. Nach dem Tod des Partners, auch in einer lang anhaltenden Phase der Trauerbewältigung werden neue Tagesstrukturen gesucht. Auch eine eigene Krankheit kann den Impuls geben, in der Gemeinschaft mit anderen nach Ablenkung zu suchen. Gemeinsam ist diesen Personen, dass unterschiedliche Lebensereignisse dazu veranlassen, das Leben neu zu strukturieren. Prägend ist auch, dass dieser Personenkreis ein starkes Engagementpotential aufweist. Sehr zeitintensiv wird sich in den Verein eingebracht, dabei kann der Verein sogar zum Lebensinhalt werden, gerade wenn der Verein zum Ersatz für das Berufsleben fungiert. Allerdings kann dieser Personenkreis nach erfolgreichem Überwinden der Krisensituation weiter auf die Suche gehen und offen für Impulse von außen sein, die ihn mittelfristig auch zu anderen Engagementfeldern führen können. 


\section{Stabilisierungsphase}

An die erfolgreich bewältigte Etablierungsphase schließt sich eine Stabilisierungsphase an (etwa 6.-10. Vereinsjahr), in der sich zeigt, ob die geschaffenen Netzwerkstrukturen tragend sind. In der Stabilisierungsphase wird deutlich, ob sich die Seniorengenossenschaft tatsächlich gefestigt hat. In dieser Phase können Impulse von außen, aber auch interne Prozesse Instabilitäten des Vereins bewirken. In der Regel kommt es in dieser Vereinsphase zum Wechsel einzelner Vorstandsposten. Gerade aufgrund der Altersstruktur ${ }^{14}$ der Mitglieder einer Seniorengenossenschaft unterliegen die Vorstandsämter einem häufigeren Wechsel, als dies in anderen eingetragenen Vereinen beobachtbar ist. ${ }^{15}$ Der Eintritt der Mitglieder in die Statuspassage ${ }^{16}$ der Hochaltrigkeit bildet in der Regel eine natürliche Grenze für eine aktive Mitgliedschaft im Vorstand. Außerdem sollte die Stabilisierungsphase von einer aktiven Mitgliederwerbung begleitet werden. So gilt es in dieser Vereinsphase besonders neue Vereinsmitglieder, denen der Charakter des „Sinn suchenden Idealisten“ anhaftet, für einen Vereinseintritt zu gewinnen, die sich dadurch auszeichnen, dass sie bereit sind, den Verein mit ihren Ideen zu prägen und auch gerne Vorstandspositionen übernehmen oder Gruppen leiten. Beispielhaft zeigt nachfolgende Abbildung die Lebenszyklusphasen einer Seniorengenossenschaft.

14 Insgesamt bestätigt die quantitative Befragung der im Bundesgebiet tätigen Seniorengenossenschaften, dass Seniorengenossenschaften Initiativen des 3. und 4. Lebensalters sind. In der Regel nimmt ein Viertel aller Mitglieder aktiv am Vereinsleben teil. Die Altersstruktur der Aktiven betrachtend ist es die Gruppe der 60-80 Jährigen, die gut zwei Drittel der Aktiven ausmacht. Das Verhältnis von Frauen zu Männern ist in der Regel 3:1.

15 Zur Wahrung einer gewissen Kontinuität sind in vielen Seniorengenossenschaften die Vorstandsposten mit je zwei Mitgliedern besetzt.

16 Definition von Statuspassagen bei: Schulz-Nieswandt 2006. 


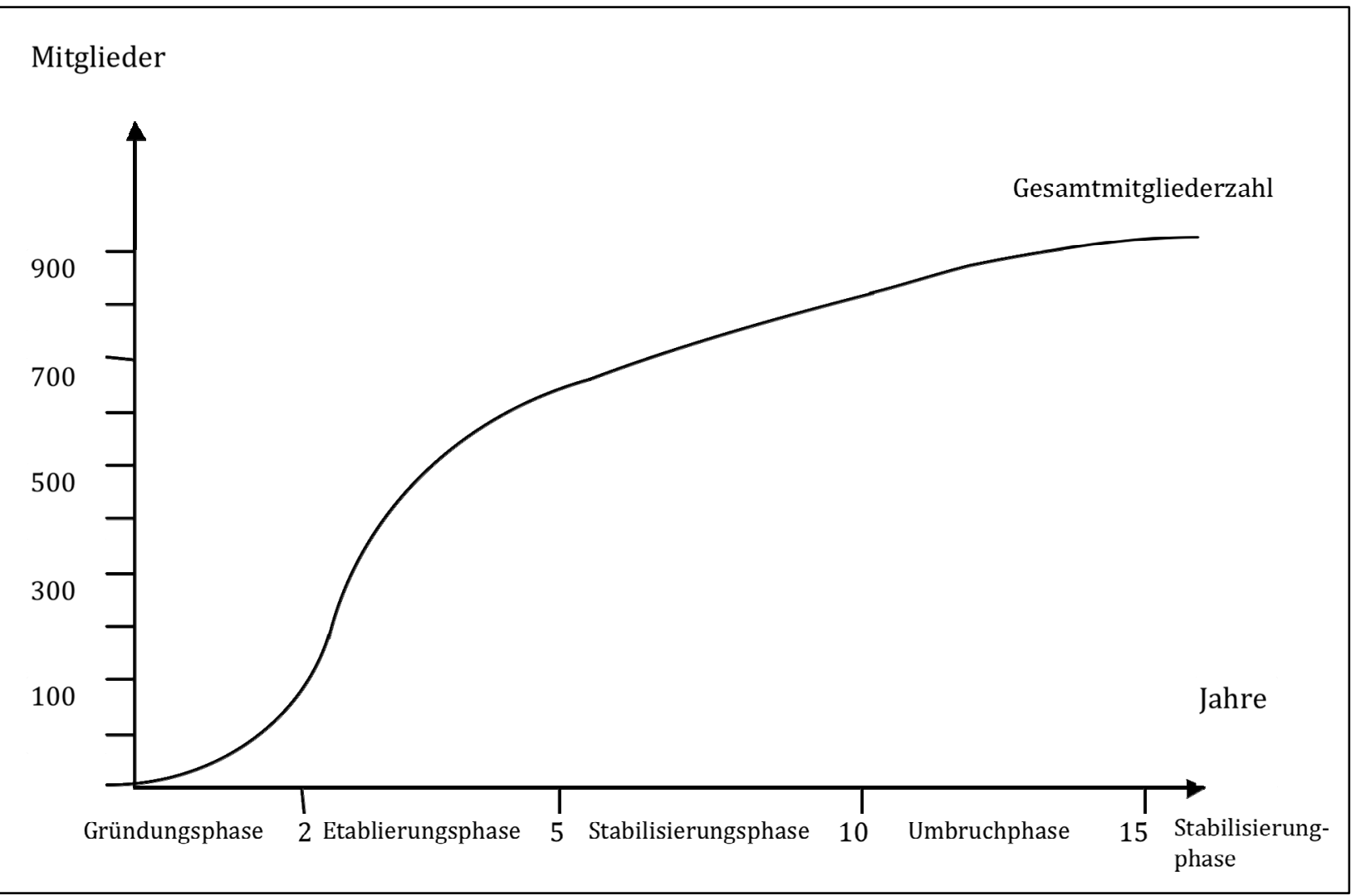

\section{Abb 1: Lebenszyklusphasen einer Seniorengenossenschaft}

Quelle: Eigene Darstellung

Zur Abbildung: In der Gründungsphase steigt die Gesamtmitgliederzahl - aktive und passive Mitgliedschaften - exponentiell an (bis ca. 100 Mitglieder). In der sich anschließenden Etablierungsphase verzeichnet der Verein sein größtes Anwachsen der Mitgliederzahl im Vereinslebenszyklus. (bis ca. 700 Mitglieder). In der Stabilisierungs- und Umbruchphase wächst der Verein weiter stetig an. Ab der 4. Phase, der Umbruchphase, hält der Verein seine Gesamtmitgliederzahl mit steigender Tendenz. Die Neuzugänge übersteigen die Austritte, da i. d. R. nur bei Tod ausgetreten wird. Ab einer Mitgliederstärke von 1.000 Mitgliedern besteht das Problem, dass sich der Charakter des Vereins dadurch verändert, dass der Verein in Untergruppen zerfällt, zwischen denen die Vernetzung schwierig wird. Bei den in Deutschland derzeit tätigen Seniorengenossenschaften variiert die Gesamtmitgliedgliederzahl zwischen 100 bis knapp 2.000 Mitglieder.

\section{Umbruchphase}

Nach zehn Vereinsjahren kann davon ausgegangen werden, dass eine Seniorengenossenschaft eine stabile Vereinsbasis gefunden hat. Diese Basis braucht der Verein, um die sich anschließende Umbruchphase (etwa 10.-15. Vereinsjahr) zu bewältigen. Die Gründergeneration tritt in das 4 . Lebensalter ${ }^{17}$ ein und zieht sich aus dem organisatorischen 
Vereinsleben zurück; bei ausreichendem Gesundheitszustand werden Veranstaltungen der Gesellung weiterhin besucht und dem Verein wird beratend treu geblieben. Entscheidend ist jedoch, die Gründergeneration kommt in die Phase der Einlösung der Zeitgutschriften. Jetzt zeigt sich, ob erfolgreich jüngere Mitglieder geworben wurden, die bereit sind, aktiv zu werden und neben dem Besuch der Gesellungsveranstaltungen auch gegenseitige Hilfen zu leisten. Die Befragung zeigt, dass die Mehrzahl der aktiven Mitglieder hofft, die gesammelten Zeitpunkte nicht einlösen zu müssen. Deutlich wird, dass bei den meisten das Geben an erster Stelle steht und das Nehmen schwer fällt. Viele Aktiven werden von einem inneren Zwang zum Geben motiviert, manche sogar getrieben. Selbst in die Situation des Annehmens von Unterstützung gekommen, fällt es ihnen schwer, im Nehmen eine Pflicht zu sehen (Krafft-Krivanec 2004). Daher kommt der Aufrechterhaltung des Kontaktes zu ehemals Aktiven eine gesonderte Bedeutung zu. Beobachtbar ist, dass ehemals Aktive zögern, bei Hilfebedarf Hilfe einzufordern. Einerseits erwarten die ehemals Aktiven Hilfe, denn sie haben vormals Hilfe geleistet, andererseits warten sie aber auch, daraufhin angesprochen zu werden. Geäußert wird, dass es einfacher ist, zu einer angebotenen Hilfe ,ja“ zu sagen, als die Forderung nach Hilfe selbst zu stellen. $\mathrm{Zu}$ beobachten ist, dass ehemals Aktive sich zurückziehen und oft nicht in der Lage sind, selbst Hilfe einzufordern. Aus dieser Konstellation kann eine Situation des Unmuts entstehen. Zur Vorbeugung dieser Situation und zur weiteren Integration ehemals Aktiver ist es daher notwendig, in dieser Phase des Generationenwechsels Koordinatoren zwischen den ehemals Aktiven und dem Verein einzurichten. In dieser Umbruchphase, in der sich der Vorstand und die aktive Kerngruppe neu formiert, sind es wieder Personen, die mit ihrem Engagement im Verein einen Lebensinhalt finden.

\section{Langfristige Stabilität von Seniorengenossenschaften}

Die ersten Seniorengenossenschaften haben derzeit erfolgreich die Umbruchphase abgeschlossen und es zeichnet sich eine erneute Stabilisierungsphase (etwa ab dem 15. Vereinsjahr) ab. Eine neue Generation von aktiven Seniorengenossenschaftlern hat die Vorstandsämter inne und die Netzwerke zu den anderen ortsansässigen Institutionen müssen gepflegt werden, Kontakte aufrechterhalten und erneuert werden. Zur zeitlichen Überlebensdauer von Seniorengenossenschaften kann festgestellt werden, dass Seniorengenossenschaften von der Lebendigkeit des Vereinslebens, d. h., von den Ideen und den Aktivitäten der Mitglieder leben. ${ }^{18}$ Der Verein muss sich ständig weiterentwickeln, nur so bekommt der Verein eine eigene Biographie. Dabei gilt es Phasen der Stagnation - im

18 Die quantitative Befragung von 50 im Bundesgebiet tätigen Seniorengenossenschaften zeigte, dass in der Regel sich ein Viertel aller Vereinsmitglieder aktiv in die Seniorengenossenschaft einbringt. Die qualitative Befragung vernachlässigt die Gruppe der dauerhaft passiven Mitglieder, die lediglich den Jahresbeitrag zahlen, ohne je an den Gesellungsveranstaltungen teilzunehmen oder gegenseitige Hilfen zu leisten. Befragt werden konnten allerdings auch ehemals Aktive, die zum Zeitpunkt der Befragung nicht mehr aktiv am Vereinsleben teilnahmen. 
Sinne von Stillstand - schnell zu überwinden. ${ }^{19}$ Es ist davon auszugehen, dass Stabilisierungsphasen und Umbruchphasen im Wechsel aufeinander folgen werden und somit die Idee Seniorengenossenschaft eine langfristige Überlebenschance bekommt. In ihren Satzungen fordern die Seniorengenossenschaften von neu beitretenden Mitgliedern zunächst das Geben. Dies geschieht nicht ohne einen gewissen sozialen Druck, denn mit der Anmeldung ist ein Beitrittszettel auszufüllen, auf dem das Neumitglied sein individuelles Angebot an Hilfen listet. Neumitglieder, die schon hilfebedürftig sind, können einen Paten angeben, der stellvertretend Punkte sammelt. So ist das System Seniorengenossenschaft per se auf generalisierte Reziprozität ausgerichtet.

Für ein langfristiges Überleben von Seniorengenossenschaften kommt neben den vereinsinternen Bedingungen den äußeren Rahmenbedingungen eine entscheidende Rolle zu. So stellen das Vereinsrecht und das Gemeinnützigkeitsrecht wichtige Faktoren für die Stabilität von Seniorengenossenschaften dar. Erfolgreich haben die ersten Vereine eine über 17jährige Vereinsbiographie geschrieben, in der sie sich auch weiterentwickelt haben und es muss neugierig abgewartet werden, wie diese in Vereinsform praktizierte, erfolgreiche Art des mitmenschlichen gegenseitigen Unterstützens weiterlebt. Denn nicht zuletzt sind Seniorengenossenschaften eingebettet in die Gesamtheit der sozialen Ordnung, die sich aufspannt aus dem Sozialen, dem Ökonomischen, der kulturellen Einbettung und dem Effizienzdenken (Schulz-Nieswandt u. a. 2006).

\section{Literaturverzeichnis}

Beyes, Timon und Urs Jäger (2005), Erforschung Multidiskursiver Organisationen. NPO-Management aus systemtheoretischer Sicht, in: Die Betriebswirtschaft 2005, Heft 6, S. 627-645.

Bundesministerium für Familie, Senioren, Frauen und Jugend 2005. Freiwilliges Engagement in Deutschland $1999-$ 2005, S. 18, Graphik Z2, Berlin.

Köstler, Ursula (2006), Seniorengenossenschaften - Stabilitätseigenschaften und Entwicklungsperspektiven, Eine empirische Studie zu Sozialgebilden des Dritten Sektors auf Grundlage der Gegenseitigkeitsökonomik, Reihe: Neue Kölner Genossenschaftswissenschaft Band 2, Münster.

Köstler, Ursula (2007), Aktivierung des Bürgers mittels Zeittauschsystemen, Seniorengenossenschaften sind mehr als nur Tauschringe, in: Zeitschrift für öffentliche und gemeinwirtschaftliche Unternehmen, Heft 4/2007, S. 390-413.

Köstler, Ursula und Frank Schulz-Nieswandt (2009), Genossenschaftliche Selbsthilfe von Senioren. Motive und Handlungsmuster bürgerschaftlichen Engagements, Stuttgart.

Krafft-Krivanec, Johanna (2004), Der Sinn des Schenkens. Vom Zwang zu geben und der Pflicht zu nehmen, Wien.

Martin, Mike und Matthias Kliegel (2005), Psychologische Grundlagen der Gerontologie, Reihe: Grundriss Gerontologie, Band 3, Stuttgart.

Mauss, Marcel (2007), Die Gabe, Frankfurt am Main.

Patulny, Roger V. und Gunnar Lind Haase Svendsen (2007), Exploring the social capital grid: bonding, bridging, qualitative, quantitative, in: International Journal of Sociology and Social Policy, vol. 27, no. 1/2, pp. 33-51.

Schmale, Ingrid und Johannes Blome-Drees (2006), Solidarische Selbsthilfe im Gesundheitssektor, in: Liberalisierung im Gesundheitswesen - Einrichtungen des Gesundheitswesens zwischen Wettbewerb und Regulierung, Schriftenreihe der Gesellschaft für öffentliche Wirtschaft, hrsg. von Günther E. Braun und Frank SchulzNieswandt, Heft 53, Baden-Baden, S. 111-131.

Schulz-Nieswandt, Frank (2006), Sozialpolitik und Alter, Stuttgart.

19 In Hessen übernimmt die Ehrenamtsagentur des Kreises Offenbach unter der Leitung von Hans Lucas die Funktion eines Ansprechpartners, der gerade in Phasen der Stagnation den Vereinen mit neuen Impulsen zur Seite steht. 


\section{Lebenszyklus}

Schulz-Nieswandt, Frank (2006a), Die Unbedingtheit der Gabeethik und die Profanität der Gegenseitigkeitsökonomik. Die genossenschaftliche Betriebsform als Entfaltungskontext der menschlichen Persönlichkeit im Lichte einer Form-Inhalts-Metaphysik, in: Zur Relevanz des genossenschaftlichen Selbsthilfegedankens, hrsg. von Hans Jürgen Rösner und Frank Schulz-Nieswandt, Reihe: Neue Kölner Genossenschaftswissenschaft, Münster, S. 57-92.

Schulz-Nieswandt, Frank (2008), Zur Morphologie des Dritten Sektors im Gefüge zwischen Staat, Markt und Familie. Ein Diskussionsbeitrag zur Ciriec-Studie „Die Sozialwirtschaft in der Europäischen Union“, in: Zeitschrift für öffentliche und gemeinwirtschaftliche Unternehmen, Heft 3/2008, S. 323-336.

Schulz-Nieswandt, Frank, Clarissa Kurscheid, Sang-Myung Lee, Remi Maier-Rigaud und Saskia Wölbert (2006), Zur Genese des europäischen Sozialbürgers im Lichte der neueren EU-Rechtsentwicklung, Münster, S. 6.

Wagner, Simone (2008), Lokale Tauschnetze, Wiesbaden. 